\title{
Intestinal parasitic infections in renal transplant recipients
}

\begin{abstract}
The impact of intestinal parasitic infection in renal transplant recipients requires careful consideration in the developing world. However, there have been very few studies addressing this issue in Iran. This study was conducted to determine the prevalence of intestinal parasitic infections in renal transplant recipients in Iran. Stool specimens from renal transplant recipients and control groups were obtained between June 2006 and January 2007. The samples screened for intestinal parasitic infections using direct smear, formalin-ether sedimentation, Sheather's flotation and modified Ziehl-Neelsen staining methods. Out of 150 renal transplant recipients, 33.3\% (50), and out of 225 control group, $20 \%$ (45) were infected with one or more type of intestinal parasites. The parasites detected among patients included Entamoeba coli (10.6\%), Endolimax nana (8.7\%), Giardia lamblia (7.4\%), Blastocystis spp. (4.7\%), Iodamoeba butschlii (0.7\%), Chilomastix mesnili $(0.7 \%)$ and Ascaris lumbricoides $(0.7 \%)$. Multiple infections were more common among renal transplant recipients group $(\mathrm{p}<0.05)$. This study highlights the importance of testing for intestinal parasites among Iranian renal transplant recipients. Routine examinations of stool samples for parasites would significantly benefit the renal transplant recipients by contributing to reduce severe infections.
\end{abstract}

Keywords: intestinal parasites, renal transplant recipients, prevalence.

[Braz J Infect Dis 2010;14(1):15-18] @Elsevier Editora Ltda.

\section{INTRODUCTION}

About 340 parasitic species infect more than three billion people worldwide with varying morbidity and mortality. ${ }^{1}$ Infections cause significant morbidity and mortality among immunosupressed hosts. Acquisition of infection, clinical severity, and outcome of a parasitic disease depend on innate and acquired host immunity as well as on the parasites' own response against the host when the infection is established. The incidence and prevalence of parasitic infections in transplant recipients is unknown since only a few patients are symptomatic. ${ }^{2}$ Only $5 \%$ of known humanpathogenic parasitic infections have been reported in transplant recipients. This certainly does not represent the true prevalence because only those infections that cause significant morbidity would be expected to find their way in to the literature. ${ }^{1}$ Since the use of cyclosporine has become a cornerstone in pro- phylactic immunosuppression, this syndrome has become exceedingly rare, owing to the strong parasiticidal effect of the drug against a wide range of organisms, as documented in mice and humans. ${ }^{1,3}$ The new immunosuppressive drugs used to prevent graft rejection have lead to an increase in parasitic infections in renal transplant recipients. The purpose of our study was to evaluate the prevalence of intestinal parasites in renal transplant recipients and compare with healthy individuals.

\section{MATERIAL AND METHODS}

This cross-sectional study was conducted from June 2006 to January 2007 at Nor hospital, a referral hospital for kidney transplantation in Isfahan, Iran. Stool specimens were collected from 150 renal transplant recipients and 225 non-immunosuppressed cases that were from different wards and selected randomly as the control group. These samples were examined
Authors:

Mehdi Azami ${ }^{1 *}$

Mehran Sharifi

Sayed Hossein Hejazi

Mehdi Tazhibi ${ }^{3}$

${ }^{1}$ Department of Parasitology \& Mycology, School of Medicine, Isfahan University of Medical Sciences, Isfahan, Iran. ${ }^{2}$ Department of Internal Medicine, School of Medicine, Isfahan

University of Medical Sciences, Isfahan, Iran. ${ }^{3}$ Department of Biostatistics \& Epidemiology, School of Health, Isfahan University of Medical Sciences, Isfahan, Iran.
Submitted on: 06/20/2009 Approved on: 10/30/2009

Correspondence to: Mehdi Azami, Department of Parasitology \& Mycology, School of Medicine, Isfahan University of Medical Sciences, Isfahan, Iran. Telephone number: +98-913-366 8290; E-mail:mehdi.azami@ gmail.com

This study received financial support from the Department of Internal Medicine, School of Medicine, Isfahan University of Medical sciences, Isfahan, Iran.

We declare no conflict of interest. 
microscopically following direct and formalin-ether concentration method. ${ }^{4}$ In brief, samples were collected in labeled, leak-proof, and clean plastic stool cups and brought to the laboratory immediately. Direct microscopy of the smears in a saline $(0.85 \% \mathrm{NaCl}$ solution) and Lugol's iodine was performed for the detection of ova, larvae, trophozoites, and cysts of intestinal parasites. In addition, a concentration procedure was employed that involved mixing the stool samples with formalin, treating with ether, centrifuging afterwards. The layers of ether, formalin, and debris were discarded, and the residues were used to investigate for the presence of intestinal parasites. ${ }^{4}$ Also, we used of Sheather's flotation method and modified Ziehl-Neelsen staining technique for detection of coccidian parasites.

The data was analyzed using SPSS version 13 statistical package. A comparison of the frequency of parasites between cases and controls was performed by chi-square test. Subsequently, the Wilcoxon Rank Sum test was used to compare multiple parasitic infections in cases and controls. $p$ value $<0.05$ was considered significant.

\section{RESULTS}

A total 375 fecal samples were collected for this study. 150 were renal transplant recipients with a mean age of 42 years. Of these, $104(69.4 \%)$ were males and $46(30.6 \%)$ were females (Table 1). The control group included 225 subjects (Table 1). There were no statistically significant differences between males and females in two groups ( $\mathrm{p}>0.05)$.

Intestinal parasites were detected in $33.3 \%$ of the renal transplant recipients and in $20 \%$ of the control. Table 2 shows the prevalence of intestinal parasites detected in the study subjects. No statistically significant difference in prevalence of individual parasite species was detected between cases and controls $(\mathrm{p}>0.05)$. Protozoa intestinal parasites were most common than helminthes both in renal transplant recipients $(29.4 \%$ versus $0.7 \%)$ and in controls $(19.2 \%$ versus $0.9 \%$ ) (Table 2 ), $\mathrm{p}<0.05$.
Table 3 shows the magnitude of single and multiple parasitic infections in renal transplant recipients and in controls. Multiple parasitic infections were observed in a total of 15 renal transplant recipients and 20 controls $(\mathrm{p}<0.05)$. The species of parasites most frequently seen in multiple infections in renal transplant recipients were Entamoeba coli and Blastocystis spp.

\section{DISCUSSION}

Protozoa and helminthes are among the most important pathogens that can cause infections in immunocompromised hosts. These microorganisms particularly infect individuals with impaired cellular immunity; such as those with hematological malignancies, renal or heart transplant patients, patients using high doses of corticosteroids, and patients with acquired immunodeficiency syndrome. ${ }^{5}$

In this study, we evaluated the prevalence of intestinal parasites in renal transplant recipients, comparing to healthy individuals. According to the results the overall prevalence's of either helminthes or protozoan parasites were not statistically different between two groups. This observation may agree with several reports stating that intestinal parasitic infections in immunocompromised patients depend largely on the prevalence of intestinal parasitism in the local community. ${ }^{6,7}$

Prevalence of intestinal parasites in renal transplant recipients are not known in Iran, so we had to compare our results to other studies on immunocompromised individuals such as HIV patients. Intestinal parasitic infection did not appear to be highly prevalent in our population. An intermediate to low level of prevalence was found in comparison with data from prevalence studies carried out in other regions ${ }^{8,9}$ In a recent study carried out on renal transplant recipients, the overall prevalence of intestinal parasites was $2.4 \% .^{10}$

In the present study, E. coli was the first most prevalent parasite detected in both groups, without significant difference and followed by Endolimax nana and Giardia lamblia. In a recent study in Iran, rate of infection with E. coli, E. nana

Table 1. Age and sex distribution of renal transplant recipients and controls

\begin{tabular}{|c|c|c|c|c|}
\hline \multirow{3}{*}{ Age } & \multicolumn{2}{|c|}{ Renal transplant recipients } & \multicolumn{2}{|c|}{ Controls } \\
\hline & Male & Female & Male & Female \\
\hline & No. (\%) & No. (\%) & No. (\%) & No. (\%) \\
\hline$<20$ years & $1(1)$ & $4(8.7)$ & $23(19.5)$ & $30(28)$ \\
\hline 21-35 years & $30(28.8)$ & $14(30.4)$ & $28(23.7)$ & $39(36.4)$ \\
\hline $36-50$ years & $41(39.4)$ & $10(21.7)$ & $26(22)$ & $23(21.5)$ \\
\hline $51-65$ years & $27(26)$ & $13(28.3)$ & $22(18.6)$ & $15(14)$ \\
\hline$>65$ years & $5(4.8)$ & $5(10.9)$ & $19(16.1)$ & $0(0)$ \\
\hline Total & $104(69.4)$ & $46(30.6)$ & $118(52.5)$ & $107(47.5)$ \\
\hline
\end{tabular}


Table 2. Prevalence of intestinal parasites in renal transplant recipients and controls

\begin{tabular}{lcc}
\hline Parasite species & $\begin{array}{c}\text { Renal transplant recipients }(\mathbf{n}=\mathbf{1 5 0}) \\
\text { No. (\%) }\end{array}$ & $\begin{array}{c}\text { Controls }(\mathbf{n}=\mathbf{2 2 5}) \\
\text { No. (\%) }\end{array}$ \\
\hline Entamoeba coli & $16(10.6)$ & $17(7.6)$ \\
\hline Endolimax nana & $13(8.7)$ & $15(6.7)$ \\
\hline Giardia lamblia & $11(7.4)$ & $4(1.8)$ \\
\hline Blastocystis spp. & $7(4.7)$ & $5(2.2)$ \\
\hline Iodamoeba butichilli & $1(0.7)$ & $1(0.4)$ \\
\hline Chilomastix mesnili & $1(0.7)$ & $0(0)$ \\
\hline Cryptosporidium spp. & $0(0)$ & $1(0.4)$ \\
\hline Ascaris lumbricoides & $1(0.7)$ & $0(0)$ \\
\hline Hymnolepis nana & $0(0)$ & $2(0.9)$ \\
\hline All protozoa & $49(29.4)$ & $43(19.2)$ \\
\hline All helminthes & $1(0.7)$ & $2(0.9)$ \\
\hline Total infected & $50(33.3)$ & $45(20)$ \\
\hline
\end{tabular}

Table 3. Single and multiple parasitic infections in renal transplant recipients and controls

\begin{tabular}{lccc}
\hline \multirow{2}{*}{ Subjects } & \multicolumn{3}{c}{ Parasites detected } \\
\cline { 2 - 4 } & One & Two & Three \\
No. (\%) \\
\hline Renal transplant recipients $(\mathrm{n}=150)$ & No. (\%) & $12(8)$ & $3(2)$ \\
\hline Controls $(\mathrm{n}=225)$ & $205(91.2)$ & $5(2.2)$ & $15(6.6)$ \\
\hline
\end{tabular}

and G. lamblia in HIV patients have been reported $0.16 \%$, $0 \%$ and $4.1 \%$, respectively. ${ }^{6}$ In other study carried out on renal transplant recipients in Brazil, G. lamblia was the third most prevalent parasite (3/16) and Strongyloides stercoralis was the common parasite $(11 / 16)$ in these patients. ${ }^{10}$

Cryptosporidium infection is prevalent in communities with overcrowding and low level sanitation, ${ }^{11}$ and its prevalence reaches up to $36 \%$ in certain developing countries. ${ }^{12}$ In Iran, the exact coccidian infection rates are not known and there are very few studies on cryptosporidiosis. The previous reports indicate the prevalence of this infection in diarrhea patients of $4.7 \%{ }^{13}$ and in HIV-patients of $1.5 \%{ }^{6}$ The isolation rate was low in our subject $(0.4 \%)$, compared to control group. This could be attributed to the relatively preserved immune status of our study subject.

Cyclospora cayetanensis is an opportunistic protozoan related to outbreaks, and in endemic areas, causing prolonged diarrhea in immunocompetent, as well as, in immunocompromised individuals. ${ }^{14}$ According to our study, none of investigated specimens were positive to $C$. cayetanensis infection. Recently, a study suggested a rare distribution of this parasite in Iran, with only two cases reported so far. ${ }^{15,16}$
S. stercoralis has the unique feature of transmitting from the parasitic form to the infective stage within the body, rather than emerging and forming free-living stages, causing autoinfection. ${ }^{17}$ This may lead to latent infection for an indefinite period in an immunocompetent host, but may also cause fatal hyper-disseminated infection organ transplant recipients, cancer and other immunosuppressive condition. ${ }^{18}$ In the present study, no S. stercoralis were detected in both groups. It is a fact that, the use of Cyclosporine A (CsA) has become a cornerstone in prophylactic immunosuppression among renal transplant recipients. Cyclosporine A with powerful properties of immunosuppression, acts on parasitic infections in various ways. ${ }^{19}$ There are few articles that reported CsA has reduced the incidence of strongyloidiasis in renal transplant recipients. ${ }^{1,20}$ The rate of infection with S. stercoralis in HIV-patients in $\operatorname{Iran}^{20}$ and renal transplant recipients in Brazil ${ }^{10}$ have been reported $0.2 \%$ and $68.8 \%$ respectively.

The detection of such common intestinal parasites in both patients and controls could be a reflection of the poor environmental sanitation and personal hygienic practices, which emphasize the need for intervention measures at the community level to reduce the risk factors of acquiring intestinal parasites. 
It was also evident that multiple parasitic infections were more common in renal transplant recipients than in controls, which strongly indicates the facilitated establishment of parasites in immunocompromised patients. It is very important to target these common infections while treating renal transplant recipients for opportunistic infections in developing countries like Iran.

In conclusion, the magnitude of intestinal parasitic infection was high both in renal transplant recipients and controls. Routine examination of stool samples for parasites could significantly benefit the renal transplant recipients and uninfected individuals by contributing to reduce clinical severity and improved quality of live.

\section{ACKNOWLEDGMENTS}

We are grateful to the study participants, without whom this could not have been completed. Also we thanks to Mrs. Khojandi and Mrs. Tosifiyan for all helps.

\section{REFERENCES}

1. Barsoum RS. Parasitic infections in organ transplantation. Exp Clin Transplant 2007; 2:258-67.

2. Thom K, Forrest G. Gastrointestinal infections in immunocompromised hosts. Curr Opin Gastroenterol 2006; 22:18-23.

3. Armson A, Cunningham GA, Grubb WB, Mendis AHW. Murine strongyloidiasis: the effect of cyclosporin A and thiabendazole administered singly and in combination. Int J Parasitol 1999; 25:533-5.

4. World Health Organization. Basic laboratory methods in medical parasitology. World Health Organization, Geneva, 1991.

5. Ferreira MS, Borges AS. Some aspects of protozoan infections in immunocompromised patients - A review. Mem Inst Oswaldo Cruz Rio de Janeiro 2002; 97:443-57.

6. Meamar AR, Rezaian M, Mohraz M et al. A comparative analysis of intestinal parasitic infections between HIV/AIDS patients and non-HIV infected individuals. Iranian J Parasitol $2007 ; 2: 1-6$
7. Cottee L, Rabodonirina M, Piens MA et al. Prevalence of intestinal protozoan in French patients infected with HIV. J Acquir Immune Defic Syndr 1993; 6:1424-29.

8. Getachew H, Afework K, Gemeda A et al. Intestinal parasitic infections in HIV/AIDS and HIV seronegative individuals in a teaching hospital, Ethiopia. Jpn J Infect Dis 2004; 57:41-3.

9. Mohandas K, Seghal R, Sud A, Malla N. Prevalence of intestinal parasitic pathogens in HIV-seropositive individuals in northern India. Jpn J Infect Dis 2002; 55:83-4.

10. Valar C, Keitel E, Dal-Pra RL et al. Parasitic infection in renal transplant recipients. Transplant Proc 2007; 39:460-2.

11. Brink AK, Mahe C, Watera C et al. Diarrhea, CD4 counts and enteric infections in a community-based cohort of HIV-infected adults in Uganda. J Infect 2002; 45:99-106.

12. Gomez-Morales MA, Atzori C, Ludovisi A et al. Opportunistic and non-opportunistic parasites in HIV-positive and negative patients with diarrhea in Tanzania. Trop Med Parasitol 1995; 46:109-14.

13. Azami M, Dorostkar Moghaddam D, Salehi R, Salehi M. The Identification of Cryptosporidium Species in Isfahan, Iran by PCR-RFLP Analysis of the 18S rRNA Gene. Appl Molecul Biol 2007; 5:851-6.

14. Chacin-Bonila L, Estves J, Monsalve F, Quijada L. Cyclospora cayetanensis infections among diarrheal patients from Venezoela. Am J Trop Med Hyg 2001; 63:351-4.

15. Rezaian M, Hoshyar H. Human infection with Cyclospora cayetanensis: report of a case. Hakim J 2000; 3:39-43.

16. Rezaian M, Mahbod AA, Mohraz M, Meamar AR. Cyclosporiasis report of the first case in Iran. Iranian J Infect Dis Trop Med 1996; 1:34-42.

17. Schaeffer MW, Bull JF, Gupta M et al. Strongyloides hyper infection syndrome after heart transplantation: case report and review of the literature. J Heart Lung Transplant 2004; 23:905-11.

18. Keiser PB, Nutman TB. Strongyloides stercoralis in immunocompromised population. Clin Microbiol Rev 2004, 17:208-17.

19. Chappell LH, Wastling JM. Cyclosporin A: antiparasite drug, modulator of the host-parasite relationship and immunosuppressant. Parasitol 1992; 105:25-40.

20. Zali MR, Jafari-Mehr A, Rezaian M et al. Pravalence of intestinal parasitic pathogens among HIV-positive individuals in Iran. Jpn J Infect Dis 2004; 57:268-70. 